\title{
Educação em saúde na prevenção do pé diabético na atenção primária: uma revisão integrativa
}

\author{
Health education in the prevention of diabetic foot in primary care: an integrative review \\ La educación en salud en la prevención del pie diabético en la atención primaria: una revisión \\ integrativa
}

Ariellen Martins Guerra

ORCID: https://orcid.org/0000-0001-6201-2806 Instituto de Educação Superior Vale do Parnaíba, Brasil E-mail: ariellenmartins1@ hotmail.com

Guilherme Araújo da Silva

ORCID: https://orcid.org/0000-0001-5579-4459 Instituto de Educação Superior Vale do Parnaíba, Brasil E-mail: guilherme11araujo@gmail.com

Antônio Victor de Oliveira Machado

ORCID: https://orcid.org/0000-0003-0016-5959 Instituto de Educação Superior Vale do Parnaíba, Brasil E-mail: victor2711@live.com

Filipe Levy Leite Visgueira

ORCID: https://orcid.org/0000-0002-0849-6835

Centro Universitário Uninovafapi, Brasil

E-mail: visgueira.filipe@gmail.com

Francisco Hildebrando Moreira de Oliveira Filho

ORCID: https://orcid.org/0000-0002-8362-4374 Instituto de Educação Superior Vale do Parnaíba, Brasil E-mail: bambam.igt@gmail.com

Maria das Graças Resende da Silva Neta

ORCID: https://orcid.org/0000-0002-0945-1751 Instituto de Educação Superior Vale do Parnaíba, Brasil E-mail: gracinharesende@ outlook.com

Lucas Benjamim Pereira Farias ORCID: https://orcid.org/0000-0002-9133-1475 Instituto de Educação Superior Vale do Parnaíba, Brasil

E-mail: lucasbenjamim35@hotmail.com

Célia Barros de Sousa Marques ORCID: https://orcid.org/0000-0001-9309-1893 Instituto de Educação Superior Vale do Parnaíba, Brasil E-mail: celiamarques_@outlook.com

Raquel Araújo Nogueira

ORCID: https://orcid.org/0000-0001-8535-6847 Instituto de Educação Superior Vale do Parnaíba, Brasil E-mail: raquelaraujo852@gmail.com

Ana Clara Correia Gomes

ORCID: https://orcid.org/0000-0003-2463-0720 Instituto de Educação Superior Vale do Parnaíba, Brasil E-mail: clarinhacorreiaanaclaracorreia@hotmail.com

David Danisio Silva de Freitas

ORCID: https://orcid.org/0000-0003-4003-1971 Instituto de Educação Superior Vale do Parnaíba, Brasil

E-mail: fullbringboy@gmail.com

Francisco de Nojosa Costa Neto ORCID: https://orcid.org/0000-0001-8721-5087 Instituto de Educação Superior Vale do Parnaíba, Brasil

E-mail: netotoc99@hotmail.com

Alyne de Araújo Paiva

ORCID: https://orcid.org/0000-0002-1950-0887 Instituto de Educação Superior Vale do Parnaíba, Brasil

E-mail: alynepaivaa@icloud.com

Daniel Barbosa Lima

ORCID: https://orcid.org/0000-0002-0801-4410 Instituto de Educação Superior Vale do Parnaíba, Brasil E-mail: danielbarbosa179@gmail.com 


\author{
Gabrielle Agostinho Rolim Marques \\ ORCID: http://orcid.org/ 0000-0002-0036-8126 \\ Instituto de Educação Superior Vale do Parnaíba, Brasil \\ E-mail: gabrielle.marques@iesvap.edu.br
}

\begin{abstract}
Resumo
Introdução: Com a criação do Sistema Único de Saúde (SUS), por meio da Atenção Primária a Saúde (APS), a população tem acesso a um ambiente de saúde multidisciplinar, capaz de atender as mais variadas demandas, principalmente no que tange as Doenças Crônicas Não Transmissíveis (DCNT). Nesse contexto, as complicações decorrentes do pé diabético necessitam de atenção especial, visto que ainda possuem alta incidência e suas complicações afetam de forma significativa a vida dos pacientes. Objetivos: Analisar o impacto das ações realizadas para atenção ao paciente com pé diabético pela APS, enfatizando a importância da capacitação dos profissionais de saúde. Metodologia: Trata-se de uma revisão integrativa de abordagem qualitativa, sendo utilizadas as bases de dados BVS, SciELO e MEDLINE, utilizando-se os descritores "profissionais da saúde"; "educação em saúde", "pé diabético" e "atenção primária". Dentre os critérios de inclusão, estão artigos científicos nos idiomas português, inglês e espanhol publicados entre os anos de 2010 a 2021, como critério de exclusão tivemos artigos duplicados e que não conseguiam responder o problema e objetivos da temática. Foram encontrados 58 artigos, sendo que ao final foram analisados 8 artigos. Considerações Finais: Após a realização do presente trabalho, conclui-se que o desenvolvimento de atividades que englobam a educação em saúde voltada aos profissionais da saúde na APS apresenta impactos positivos na prevenção e manejo de pacientes com o pé diabético. No entanto, revelam-se muitas barreiras que dificultam esse processo, sendo necessário maiores investimentos humanos, financeiros e tecnológicos nessa área.
\end{abstract}

Palavras-chave: Profissionais da saúde; Educação em saúde; Pé diabético; Atenção primária.

\begin{abstract}
Introduction: With the creation of the Sistema Único de Saúde (Unified Health System) - SUS, through Primary Health Care (PHC), the population has access to a multidisciplinary health environment, capable of meeting the most varied demands, especially with regard to Diseases Non-Communicable Chronicles (DCNT). In this context, complications arising from the diabetic foot need special attention, as they still have a high incidence and its complications significantly affect the lives of patients. Objectives: To analyze the impact of actions taken to care for patients with diabetic foot by the PHC, emphasizing the importance of training health professionals. Methodology: This is an integrative review with a qualitative approach, using the databases BVS, SciELO and MEDLINE, using the descriptors "health professionals"; "health education", "diabetic foot" and "primary care". Among the inclusion criteria are scientific articles in Portuguese, English and Spanish published between the years 2010 to 2021, as an exclusion criterion we had duplicate articles that were unable to answer the problem and objectives of the theme. 58 articles were found, and in the end 8 articles were analyzed. Final Considerations: After carrying out this study, we concluded that the development of activities that encompass health education aimed at health professionals in PHC has positive impacts on the prevention and management of patients with diabetic foot. However, there are many barriers that hinder this process, requiring greater human, financial and technological investments in this area.
\end{abstract}

Keywords: Health occupations; Health education; Diabetic foot; Primary health care.

\title{
Resumen
}

Introducción: Con la creación del Sistema Único de Salud (SUS), a través de la Atención Primaria de Salud (APS), la población accede a un entorno de salud multidisciplinario, capaz de atender las más variadas demandas, especialmente en lo que se refiere a Enfermedades Crónicas No Transmisibles (DCNT). En este contexto, las complicaciones derivadas del pie diabético requieren una atención especial, ya que aún tienen una alta incidencia y sus complicaciones afectan significativamente la vida de los pacientes. Objetivos: Analizar el impacto de las acciones realizadas por la APS en la atención al paciente con pie diabético, destacando la importancia de la formación de los profesionales de la salud. Metodología: Se trata de una revisión integradora con enfoque cualitativo, utilizando las bases de datos BVS, SciELO y MEDLINE, utilizando los descriptores "profesionales de la salud"; "Educación para la salud", "pie diabético" y "atención primaria". Entre los criterios de inclusión se encuentran artículos científicos en portugués, inglés y español publicados entre los años 2010 a 2021, como criterio de exclusión tuvimos artículos duplicados que no pudieron dar respuesta a la problemática y objetivos del tema. Se encontraron 58 artículos y al final se analizaron 8 artículos. Consideraciones finales: Tras la realización de este estudio, concluimos que el desarrollo de actividades que engloben la educación en salud dirigidas a los profesionales de la salud en la APS tiene impactos positivos en la prevención y manejo de los pacientes con pie diabético. Sin embargo, existen muchas barreras que dificultan este proceso, requiriendo mayores inversiones humanas, financieras y tecnológicas en esta área.

Palabras clave: Profesionales de salud; Educación en Salud; Pie diabético; Atención primaria de salud.

\section{Introdução}

Em 1988 o Sistema Único de Saúde (SUS) foi garantido pela Constituição Federal Brasileira, que determina que é dever do Estado garantir saúde a toda a população (Mendes, 2016). Com o surgimento desse sistema unificaram-se todos os 
serviços, desde a esfera municipal, estadual, federal até as redes privadas (quando há convênios ou contratações). Com isso, em 1990 surgiu a Lei n. 8.080/90 que sistematiza a respeito dos requisitos necessários para promoção, proteção e recuperação da saúde, além de organizar e promover o funcionamento de atividades propostas pelo SUS. Assim, institui-se um sistema de saúde que começa a ofertar uma assistência longitudinal e contínua, abordando tanto a prevenção quanto a recuperação em saúde (Da Silva, 2021).

Em face disso, para melhor organização do SUS, foi proposto a elaboração e aplicação de uma rede em que os serviços são estruturados de forma regionalizada e hierarquizada, com intuito de abranger as problemáticas de acordo com a demanda de cada região (Miranda, 2017). Dessa forma, para ser entendida a organização desse sistema, foi proposto um modelo de serviço, divido em unidades que prestam atendimento de saúde, estando inclusos estabelecimentos, redes e sistemas (Santos, 2017). Embora exista um modelo de hierarquização, organizado em atenção básica, atenção de média complexidade e de alta complexidade, não existe uma sobreposição entre os serviços, uma vez que apresentam comunicação entre esses eixos de forma integrada (Mendes, 2020).

Nesse contexto, no modelo adotado pelo SUS, a atenção básica à saúde também é chamada de atenção primária à saúde (APS) e é o primeiro nível de atenção, conforme mostra o Decreto n. 648, de 28 de março de 2006, do Ministério da Saúde (Sellera, 2020). Assim, a atenção primária inclui uma série de procedimentos menos rebuscados caracterizados por tecnologia de baixa densidade. A APS é entendida como capaz de resolver os problemas de saúde mais comuns na comunidade (Massuda, 2020). Porém, sua organização, desenvolvimento e aplicação nem sempre são fáceis de entender, exigindo um conhecimento profundo da realidade da população assistida. Dessa forma, como infraestrutura necessária para a atenção primária à saúde, destaca-se o setor de saúde básica, independentemente de haver ou não o Estratégia Saúde da Família (ESF) (Bousquat, 2019).

A APS tem chamado a atenção para o debate sobre o processo de serviços voltado para o combate às doenças crônicas não transmissíveis (DCNTs) (De Medeiros, 2021). Até o momento, a APS tem a Estratégia Saúde da Família (ESF) como principal forma de atuação, no entanto devido, a maioria das vezes o paciente apresentar resistência em buscar atendimento, o sistema não consegue oferecer atendimento integral e contínuo para algumas pessoas com DCNTs (BECKER, 2018). Em consequência disso, em 2015, no Brasil, as DCNTs foram responsáveis por 75\% de todas as mortes, das quais os principais grupos de etiologias são: doenças do aparelho circulatório, câncer, doenças respiratórias crônicas e diabetes (Malta, 2017). Sabe-se ainda que esses quatro grupos compartilham os principais fatores de risco para doenças crônicas: alimentação inadequada, tabagismo, sedentarismo e consumo abusivo de álcool (Rocha-Brischiliari, 2019).

Em face disso, o diabetes mellitus tipo 2 (DM2) é visto como um problema de saúde pública, sendo uma doença que atinge a população em geral (Brutti, 2019). O DM2 é uma das doenças crônicas mais comuns, respondendo por 90\% dos casos, e é descrito como uma patologia silenciosa (Câmara, 2019). Assim, devido às altas taxas de internação e incapacidade física por complicações macrovasculares e microvasculares, o DM2 é uma das doenças que mais demandam serviços de saúde, o que faz com que o sistema de saúde de todos os países fique sobrecarregado, independentemente de seu desenvolvimento econômico (Souza Junior, 2019). Dessa forma, o principal objetivo da APS é controlar a glicemia sanguínea em pacientes DM2, reduzir a incidência e morbimortalidade de complicações cardiovasculares, bem como melhorar a qualidade de vida, estimular e motivar os pacientes a cuidarem de si mesmos (Fonseca, 2019).

Nesse sentido, a identificação de fatores de risco, sinais de acometimento de órgãos-alvo, sintomas de hiperglicemia ou hipoglicemia, a forma como os pacientes perceberem sua doença e como aderem ao tratamento afeta diretamente o monitoramento domiciliar da glicose (Nunes, 2019). Sabe-se que existem muitas complicações sérias e onerosas que afetam as pessoas com DM2, como doenças cardíacas, problemas renais, cegueira e complicações nos pés, também conhecido como pé diabético (De Sousa, 2019). Nesse interim, o pé diabético é responsável por 40\% a 70\% de todas as amputações de membros 
inferiores que estão relacionadas ao diabetes, e aproximadamente $85 \%$ de todas as úlceras nos pés ocorrem antes da amputação diabética. Dessa forma, o cuidado com os pés é fundamental para reduzir o número de casos prejudicados por neuropatia e perda da sensibilidade periférica ao toque, calor e dor, que podem levar a lesões complexas que, se não tratadas, podem evoluir com amputação (Do Nascimento, 2019).

Assim, sabe-se que cerca de $85 \%$ dos problemas que são ocasionados pelo pé diabético podem ser evitados, principalmente por medidas preventivas, tais como: avaliação regular dos pés por profissionais de saúde, associado ao autocuidado do paciente (Ramirez-Perdomo, 2019). Nesse interim, é de suma importância que os profissionais sejam capacitados para realizar uma avaliação clínica eficaz em pacientes com lesões aparente nos pés, decorrentes da DM2 (Cubas, 2013).

Dessa maneira, o presente estudo tem como objetivo identificar o nível de capacitação dos profissionais de saúde na APS quanto ao manejo e abordagem do pé diabético, bem como analisar o nível de impacto na saúde desses pacientes caso o manejo seja realizado de forma adequada ou não.

\section{Metodologia}

Trata-se de uma revisão integrativa de abordagem qualitativa, que tem como intuito identificar e sintetizar pesquisas publicadas em base de dados, ou seja, apresenta conclusões a respeito de pesquisas anteriormente relatadas. Dessa maneira, a revisa integrativa engloba todos os estudos associados com a pergunta norteadora que direciona a busca deste estudo, assim os dados são sintetizados e comparados, permitindo o desenvolvimento de conclusões acerca da problemática do estudo (Crossetti, 2012).

Dessa forma, para desenvolver a presente revisão, foi necessário realizar as seguintes etapas: estabelecer hipóteses, objetivos e tema do trabalho; estabelecer os descritores de buscas nas bases de dados; estabelecer os critérios de inclusão e exclusão para selecionar a amostra; definir os dados que serão retirados dos artigos que foram selecionados; analisar os resultados obtidos; discutir e apresentar os resultados: e, finalmente, apresentar a revisão.

Dentro da Prática Baseada em Evidências (PBE), a estratégia PICo surge com um acrômio para Paciente, Intervenção, Comparação e "Outcomes" (desfecho), sendo componentes essenciais para construção da pergunta norteadora do estudo e elaboração da pesquisa. Assim, utilizando-se da estratégia PICo, a pergunta norteadora do presente trabalho é: Como é feita a educação em saúde sobre o pé diabético para os profissionais da saúde na atenção primária?

Para a busca de artigos nas bases de dados, foram utilizadas: a BVS (Biblioteca Virtual em Saúde), o SCIELO (Scientific Electronic Library Online) e o MEDLINE (Medical Literature Analysis and Retrieval Sistem on-line). Dessa maneira, a busca foi realizada de maneira interdependente por três pesquisadores, por meio dos descritores controlados disponíveis nos Descritores em Ciência da Saúde (DeCS) e seus correspondentes em português e inglês, assim foram definidos os descritores de busca: (profissionais da saúde) AND (educação em saúde) AND (pé diabético) AND (atenção primária).

Como critérios de inclusão das publicações foram definidos: artigos publicados em português, espanhol e inglês, presentes nas bases de dados pré-estabelecidas, no período de 2010-2021; artigos que abordem sobre a importância e sobre como desenvolver a educação em saúde relacionada ao pé diabético; artigos que possuam procedimentos, intervenções e ações que estejam vinculadas ao tema central do estudo. Dentre os critérios de exclusão, estão: artigos duplicados, manuais, teses e dissertações.

A partir da busca com os descritores, nas bases de dados, foram obtidos 58 artigos. Após uma avaliação inicial, através dos resumos, verificou-se que 20 estavam no critério de exclusão temporal, 3 no critério de exclusão dos idiomas escolhidos e 3 artigos duplicados nos bancos de dados, assim, 26 estudos foram excluídos. Após essa exclusão, foi possível selecionar 32 estudos para realizar leitura e avaliação, sendo que destes, apenas 8 foram selecionados para elaborar o resultado 
dessa revisão integrativa. A Figura 1 apresenta o fluxograma que contempla o processo de seleção dos artigos que constituem este artigo.

Figura 1: Fluxograma de amostragem para seleção de artigos para composição da revisão integrativa. Parnaíba, 2021.

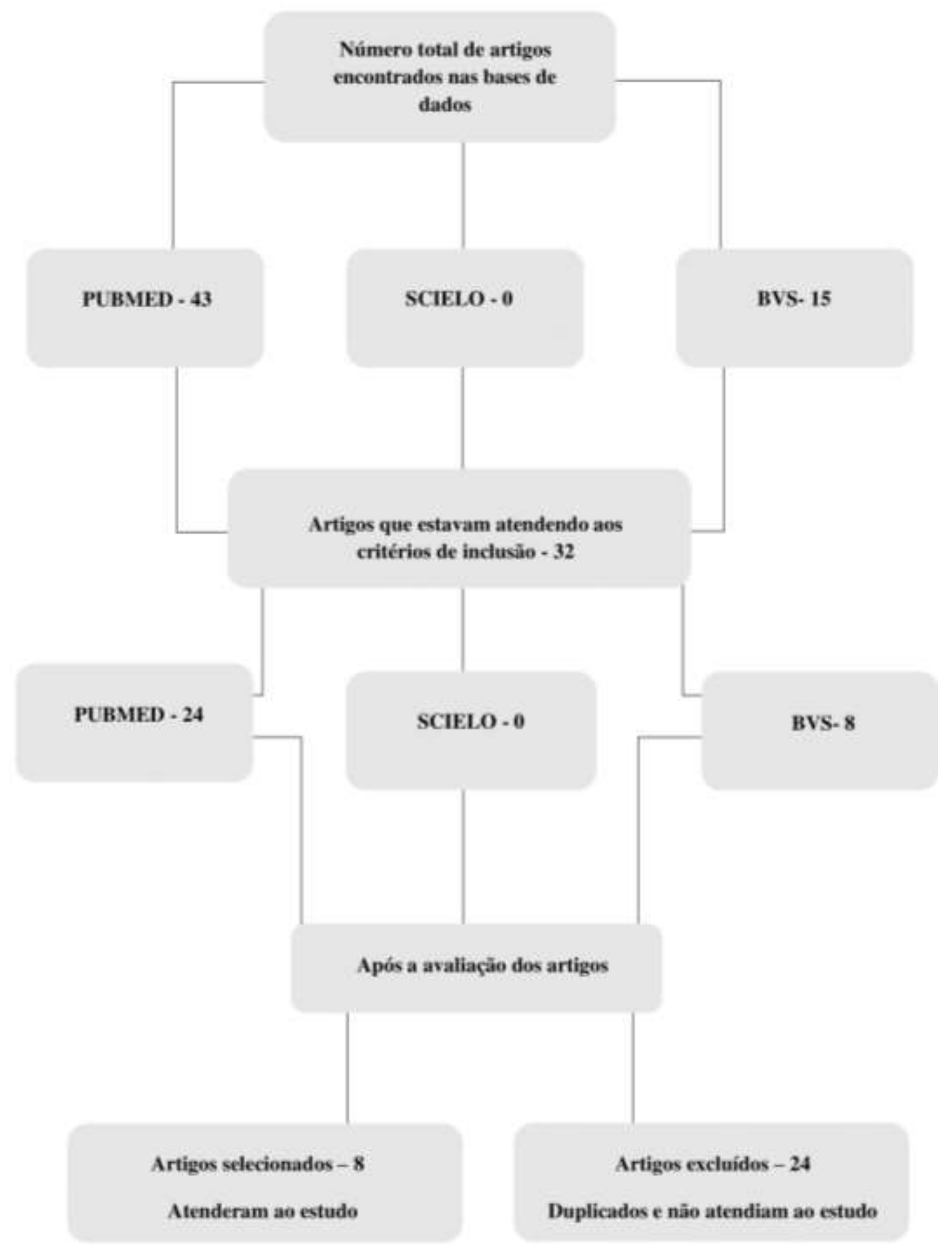

Fonte: Autores (2021).

A análise dos dados foi realizada de forma descritiva e os estudos foram reunidos a fim de avaliar se a temática e os resultados de cada um deles se encaixam nos objetos do presente estudo. 


\section{Resultados e Discussão}

Foram analisados 08 estudos (6 localizados na PUBMED, 1 na MEDLINE e 1 na BVS) sendo todos artigos de periódicos. Com relação à fonte, cada estudo foi publicado nos seguintes periódicos: Journal of foot and ankle research; Journal of clinical nursing; The Diabetes Educator; NZ Med J; BMJ open quality; Australian journal of primary health; Journal of wound care; Revista Cuidarte; BMC health services research.

Os estudos selecionados encontram-se sumarizados no Quadros 1, referente à temáticas educação em saúde na prevenção do pé diabético na APS, voltado aos profissionais da saúde.

Quadro 1: Análise de resultados dos artigos inclusos no presente estudo. Parnaíba, 2021.

\begin{tabular}{|c|c|c|c|c|c|}
\hline $\mathrm{N}^{\mathrm{o}}$ & $\begin{array}{l}\text { Base de } \\
\text { Dados }\end{array}$ & Título & Autor, Ano & $\begin{array}{l}\text { Natureza } \\
\text { do estudo }\end{array}$ & Resultados \\
\hline 1 & PUBMED & $\begin{array}{c}\text { Organizational changes in } \\
\text { diabetic foot care practices for } \\
\text { patients at low and moderate risk } \\
\text { after implementing a } \\
\text { comprehensive foot care program } \\
\text { in Alberta, Canada }\end{array}$ & $\begin{array}{l}\text { Catherine B } \\
\text { Chan et al., } \\
2020 .\end{array}$ & Transversal & $\begin{array}{c}\text { Demonstrou que a prática da educação em saúde, } \\
\text { associada ao incentivo do raciocínio clínico de manejo do } \\
\text { pé diabético, reduz significamente as complicações de tal } \\
\text { patologia. }\end{array}$ \\
\hline 2 & PUBMED & $\begin{array}{l}\text { Implementation of the Champions } \\
\text { for Skin Integrity model to } \\
\text { improve leg and foot ulcer care in } \\
\text { the primary healthcare setting }\end{array}$ & $\begin{array}{l}\text { Chrisina N } \\
\text { Parker et al., } \\
2019 .\end{array}$ & $\begin{array}{l}\text { Pré-teste, } \\
\text { pós-teste } \\
\text { não } \\
\text { equivalente }\end{array}$ & $\begin{array}{l}\text { Promoveu um workshop com a presença de oficinas para } \\
\text { profissionais de saúde da Atenção Primária, demonstrando } \\
\text { que o modelo de abordagem apresentado provocou uma } \\
\text { mudança positiva no tratamento, avaliação e prevenção de } \\
\text { complicação do pé diabético. }\end{array}$ \\
\hline 3 & PUBMED & $\begin{array}{l}\text { Improved foot management of } \\
\text { people with diabetes by primary } \\
\text { healthcare nurses in Auckland, } \\
\text { New Zealand }\end{array}$ & $\begin{array}{l}\text { Barbara Daly } \\
\text { et al.,2020. }\end{array}$ & Transversal & $\begin{array}{c}\text { Investigou que os enfermeiros aumentaram } \\
\text { significativamente seu papel no atendimento de pacientes } \\
\text { com diabetes na última década, aumentando o número de } \\
\text { exames dos pés e fornecendo educação adequada sobre } \\
\text { cuidados com os pés. }\end{array}$ \\
\hline 4 & PUBMED & $\begin{array}{l}\text { Increasing identification of foot at } \\
\text { risk of complications in patients } \\
\text { with diabetes: a quality } \\
\text { improvement project in an urban } \\
\text { primary health centre in India }\end{array}$ & $\begin{array}{l}\text { Abha } \\
\text { Mehndiratta } \\
\text { et al.,2020. }\end{array}$ & Transversal & $\begin{array}{l}\text { Padronizou o prontuário eletrônico na unidade abordada, } \\
\text { associando treinamento aos profissionais de saúde na } \\
\text { abordagem do pé diabético, provocando assim melhorias } \\
\text { no cuidado a esses pacientes. }\end{array}$ \\
\hline 5 & PUBMED & $\begin{array}{l}\text { Barriers and enablers to } \\
\text { delivering preventative and early } \\
\text { intervention footcare to people } \\
\text { with diabetes: a scoping review of } \\
\text { healthcare professionals' } \\
\text { perceptions }\end{array}$ & $\begin{array}{l}\text { Leanne } \\
\text { Mullan et al., } \\
2020 .\end{array}$ & $\begin{array}{l}\text { Estudo de } \\
\text { escopo }\end{array}$ & $\begin{array}{l}\text { Listou as barreiras e os fatores facilitadores relacionados } \\
\text { com o cuidado ao paciente com pé diabético. }\end{array}$ \\
\hline 6 & PUBMED & $\begin{array}{l}\text { Improving rural and remote } \\
\text { practitioners' knowledge of the } \\
\text { diabetic foot: findings from an } \\
\text { educational intervention. }\end{array}$ & $\begin{array}{l}\text { Deborah E } \\
\text { Schoen et } \\
\text { al.,2016. }\end{array}$ & $\begin{array}{l}\text { Quase } \\
\text { experiment } \\
\text { al de pré e } \\
\text { pós teste }\end{array}$ & $\begin{array}{c}\text { Improving rural and remote practitioners' knowledge of } \\
\text { the diabetic foot: findings from an educational } \\
\text { intervention. }\end{array}$ \\
\hline 7 & BVS & $\begin{array}{l}\text { Elaboração e validação de um } \\
\text { álbum seriado para prevenção do } \\
\text { pé diabético }\end{array}$ & $\begin{array}{l}\text { Maria } \\
\text { Auxiliadora } \\
\text { Aguiar } \\
\text { Chaves et } \\
\text { al.,2021. } \\
\end{array}$ & $\begin{array}{l}\text { Estudo } \\
\text { metodológi } \\
\text { co }\end{array}$ & $\begin{array}{l}\text { Elaboração e validação de um álbum seriado para } \\
\text { prevenção do pé diabético. }\end{array}$ \\
\hline 8 & MEDLINE & $\begin{array}{l}\text { Telemedicine in diabetes foot } \\
\text { care delivery: health care } \\
\text { professionals' experience. }\end{array}$ & $\begin{array}{l}\text { Beate- } \\
\text { Christin Hope } \\
\text { Kolltveit et } \\
\text { al.,2016. }\end{array}$ & $\begin{array}{l}\text { Descrição } \\
\text { interpretati } \\
\text { va indutiva }\end{array}$ & $\begin{array}{l}\text { O artigo direciona para os novos métodos de ensino, como } \\
\text { a telemedicina voltada para a prevenção e rastreio do pé } \\
\text { diabético para profissionais da enfermagem. Os resultados } \\
\text { indicaram que o uso de uma intervenção de telemedicina } \\
\text { permitiu aos profissionais de saúde participantes abordar } \\
\text { seus pacientes com úlcera de pé diabético com mais } \\
\text { conhecimento, melhores habilidades de avaliação de } \\
\text { feridas e maior confiança. Além disso, agilizou a } \\
\text { comunicação entre os níveis de atenção à saúde e ajudou a } \\
\text { ver os pacientes de forma mais holística. }\end{array}$ \\
\hline
\end{tabular}

Fonte: Autores (2021).

O diabetes e suas complicações podem ser considerados epidemias mundiais silenciosas, afetando em média 382 milhões de pessoas no mundo segundo Fonseca (2019). Portanto, é necessário discutir sobre as desigualdades de acesso à educação em saúde e consequentemente ao tratamento dessas patologias. Visto que, a promoção da educação continuada para os profissionais de saúde que lidam diariamente com essa temática é essencial para identificar estratégias que facilitem o 
acompanhamento e cuidado de portadores de diabetes e suas complicações.

A partir da leitura e da análise dos estudos, surgiram três núcleos temáticos: núcleo 1: Educação em saúde no manejo do pé diabético; núcleo 2: Recursos que auxiliam na abordagem do pé diabético e núcleo 3: Fatores facilitadores/barreiras presentes no cuidado do paciente com pé diabético.

\section{Núcleo 1: Educação em saúde no manejo do pé diabético}

De acordo com os estudos de Chan (2020), destaca-se que as complicações decorrentes do pé diabético comprometem, de forma significante, a vida dos pacientes acometidos por tal condição, sendo necessárias medidas de rastreio, triagem e manejo multidisciplinar para diminuir os impactos decorrentes dessa patologia. Assim, ressalta-se que a disponibilização de recursos de mídias, principalmente em formato de vídeos, se torna essencial para capacitar os profissionais em saúde da APS, na abordagem clínica dos pacientes com pé diabético, uma vez que por meio da visualização da correta avaliação dessa condição clínica, os profissionais tornam-se mais aptos no atendimento a esses pacientes. Dessa maneira, podese afirmar que a utilização dessas metodologias ativas auxiliam os profissionais a desenvolverem um melhor desempenho na realização da abordagem ao paciente com pé diabético.

Nesse sentido, de encontro com Chan (2020), Daly (2020) traz uma discussão sobre a relação do cuidado e da educação em saúde sobre o pé diabético, demonstrando que após uma capacitação de 20 horas sobre o manejo de pacientes com úlceras nos pés, junto aos profissionais de enfermagem, chegou à conclusão que há um aumento significativo na capacidade desses profissionais em relatar o exame rotineiro desses pacientes, bem como fornecer educação em saúde, que de acordo com as estatísticas dos resultados, traz maior adesão do enfermo no cuidado à sua condição patológica. Assim, pode-se notar que a capacitação dos profissionais de saúde, associado ao uso de metodologias de ensino inovadoras, trazem aos mesmos maior capacidade de prevenir e intervir nas complicações decorrer do pé diabético.

\section{Núcleo 2: Recursos que auxiliam na abordagem do pé diabético}

Os estudos de Parker (2019) apontam que o protocolo clínico baseado em evidências no que tange o atendimento ao paciente com úlceras nos pés, chamado de modelo "Champions for Skin Integrity", quando associado à workshops de treinamento, trazem aos profissionais da saúde mudança positiva no tratamento, avaliação e prevenção do pé diabético, uma vez que estes trabalhadores têm um aumento no nível de confiança na capacidade de avaliar, manejar e prevenir todos os tipos de úlceras nos membros inferiores. Dessa forma, percebe-se que a utilização de protocolos sistematizados possibilitam ao profissional de saúde desenvolver uma sequência lógica da avaliação clínica do pé diabético, permitido que esses profissionais consigam atender os pacientes de maneira adequada.

Em consonância com Parker (2019), Mehndiratta (2020) informa que a maioria das amputações de pés podem ser evitada em pessoas com diabetes, uma vez que de acordo com as diretrizes, é recomendado a avaliação dos pés de uma pessoa diabética, pelo menos uma vez ao ano, entretanto, foi identificado há a falta de política clínica, treinamento e equipamento para avaliação dos pés, tornando assim maiores as possibilidades de pacientes com DM2 desenvolverem complicações decorrentes do pé diabético. Dessa forma, entende-se que o treinamento de profissionais da saúde, processos padronizados e feedback regular de dados podem melhorar o cuidado do paciente com pé diabético. Além disso, para o melhor acompanhamento do paciente, se faz necessário um cuidado integral e continuo, na qual os indicadores de qualidade em registros eletrônicos auxiliam a monitorar a comorbidade, assim possibilitando analisar a evolução do paciente e prevenindo tais complicações.

Nesse sentido, Schoen (2016) discorre a respeito do conhecimento dos profissionais de saúde sobre diretrizes nacionais para avaliação do pé diabético e da sua estratificação de risco. Dessa maneira, o estudo avaliou o conhecimento desses trabalhadores sobre o pé diabético, a disponibilidade de equipamentos e a oferta de educação sobre a patologia em 
unidades de APS, com o intuito de analisar a eficácia dos programas de educação e treinamento sobre o pé diabético para profissionais de saúde. Portanto, é notório que a ausência de treinamentos junto a esses trabalhadores traz impacto direto na estratificação de risco dos pacientes com essa condição clínica, trazendo assim mais prejuízos ao enfermo, já que a rápida identificação do problema possibilita um melhor prognóstico.

Já Kolltveit (2016) desenvolveu um ensaio clínico randomizado visando averiguar o manejo de pacientes com ulcerações no pé diabético na APS com o auxílio de profissionais especializados por meio da telemedicina. Dessa maneira, para a elaboração do estudo utilizou uma amostragem com profissionais da saúde, englobando diversas aéreas como médicos, enfermeiros e podólogos, na qual os profissionais utilizaram uma plataforma de telemedicina interativa onde comparava imagens de úlceras nos pés diabéticos presentes na internet e imagens enviadas pelo celular, auxiliando na avaliação e tratamento das ulcerações bem como facilitando a comunicação dos profissionais sobre esse assunto. Por conseguinte, observase que o uso de telemedicina na avaliação das ulcerações em pacientes com pé diabético pode ter resultados relevantes, uma vez que a utilização dessa metodologia traz mais conhecimento e maiores habilidades na avaliação das ulcerações, documentação mais qualificada, bem como aprimoramento e simplificação da comunicação entre os profissionais da APS e os especializados.

Dessa forma, correlacionando o recurso que Kolltveit (2016) elencou que pode auxiliar no manejo do pé diabético, Chaves (2021) traz em seus estudos a apresentação de um álbum seriado de autoria própria, com informações para a prevenção do pé diabético, de forma que participaram deste estudo sete especialistas com experiência em educação em saúde, tecnologia educacional e/ou diabetes. Os pesquisadores desenvolveram uma ferramenta para avaliar o desenvolvimento do álbum, relacionando objetivos, propósitos ou metas, estrutura e apresentação, e por fim o grau de significação do material. A partir da criação desse recurso, percebeu-se que a ferramenta desenvolvida é eficaz na educação e prevenção das complicações do pé diabético, e mostrou-se como uma ferramenta educativa que pode promover a autonomia dos profissionais de saúde e dos diabéticos no cuidado, tratamento e prevenção do pé diabético. Com isso, é perceptível que utilizar ferramentas e recursos para auxiliar o profissional da saúde no atendimento ao paciente com pé diabético, traz mudanças significativas no rastreio, prevenção e promoção a saúde, uma vez que esses instrumentos facilitam a abordagem e o seguimento terapêutico.

\section{Núcleo 3: Fatores facilitadores/barreiras presentes no cuidado do paciente com pé diabético.}

Mullan (2020) aponta nos seus trabalhos que há empecilhos e aspectos facilitadores para oferecer um cuidado adequado aos pacientes com ulcerações no pé diabético segundo os profissionais da saúde. Dessa forma, de acordo com o estudo apresentado, entre os fatores que dificultam esse cuidado estão: componentes geográficos, como por exemplo, pacientes que habitam em regiões mais distantes na qual tem dificuldade de acesso aos serviços de saúde; ausência de organização do cuidado entre os serviços, na qual o serviço especializado não é realizado de forma descentralizada, sem conseguir abranger toda a população com problemas relacionados aos pés diabéticos. Além disso, ainda há barreiras envolvendo componentes administrativos e comunicativos, que dificultam a comunicação intrínseca na relação multiprofissional no paciente com polineuropatia diabética. Ademais, Mullan (2020) cita que entre esses componentes estão os sistemas de saúde implantados nas diferentes regiões. Assim, também vale ressaltar, que os recursos implantados em cada APS variam, conforme citado por Kolltveit (2016), Chaves (2021) e Schoen (2016).

Dessa forma, devido a diferença desses meios surge uma dificuldade de comunicação e fornecimento do cuidado a esses pacientes, que podem estar associadas ao processo de desorganização nas diretrizes de encaminhamento e cuidado que impactam na ausência de avaliações e exames anuais específicos voltados para esses pacientes. Além disso, sabe-se que para ter um cuidado continuo e eficaz é necessário passar pelo processo de encaminhamento, no entanto quando esse processo ocorre de forma inadequada, ocorrem prejuízos a promoção do cuidado ao pé diabético. Outra barreira, seria a ausência da 
multidisciplinaridade no cuidado a esses pacientes de alto risco, pois nota-se que há uma escassez de profissionais das mais variadas aéreas especializados nesse assunto, afetando na qualidade do atendimento prestado.

Nesse sentido, nota-se que há uma barreira relacionada a escassez de recursos no terceiro núcleo, citada por Mullan (2020), que prejudica o primeiro núcleo de educação em saúde no manejo do pé diabético, e consequentemente no segundo núcleo de educação em saúde no manejo. Dentre esses recursos escassos, estão envolvidos o tempo proposto pelos profissionais ao atendimento a esses pacientes, bem como recursos financeiros, na qual falta maiores investimentos governamentais. Por fim, a falta de especialização, educação e treinamento dos profissionais na área da saúde também são vistos como barreiras potenciais para a prestação de cuidados preventivos e intervenção precoce relacionada ao pé diabético.

Ademais, já como fatores colaboradores sugeridos para um cuidado eficaz em relação ao pé diabético, o estudo mostra a realização de um programa de cuidados com os pés voltado para os profissionais da saúde, com o intuito de aumentar a conscientização sobre o encaminhamento e o rastreamento dos pés diabético. Além disso, sugere alterações no sistema de saúde, visando implementar a padronização dos registros, protocolos de controle do diabetes, e equipe auxiliar que realizará o exame do pé antes da consulta médica, para assim fornecer um cuidado adequado a esses pacientes. Nesse sentindo, o estudo também aponta como um aspecto facilitador da realização de treinamentos relacionados as técnicas necessárias na triagem do pé diabético voltado para os profissionais da saúde mostrando assim a importância do nivelamento do cuidado em relação ao pé diabético.

\section{Conclusão}

Portanto, infere-se que a contribuição no treinamento dos profissionais de saúde da APS, associados aos recursos disponibilizados, auxiliam no rastreio, prevenção, cuidado e tratamento. Ademais, a educação em saúde é uma ponte para melhoria da qualidade do atendimento e acompanhamento de pacientes com pé diabético, sendo de suma importância que esses profissionais sejam previamente capacitados por meio das diversas metodologias existente, sejam por meio de recursos de mídia, workshops, treinamentos ou protocolos.

Como limitações do estudo, vale ressaltar que são poucas as publicações sobre os temas propostos, embora as complicações decorrentes do pé diabético tenham grande incidência e relevância na APS. Assim, esta revisão não utiliza ferramentas para avaliar a qualidade metodológica e o risco de vieses dos artigos selecionados. No entanto, a mesma pesquisa atende ao propósito de refletir sobre como a capacitação dos profissionais e escassez de recursos interfere no manejo, cuidado, atenção e prevenção do paciente com pé diabético.

Portanto, no final desta reflexão, reconhecemos o quanto há uma necessidade dos profissionais da APS utilizarem diferentes estratégias de aprendizagem sobre a abordagem do paciente com pé diabético, para assim fornecer cuidado adequado associado a educação permanente em saúde. No entanto, as reflexões aqui relatadas são a base para futuros estudos, e buscaremos aprofundar nossas reflexões e conhecimentos a fim de investigar novas sugestões de métodos construtivos a serem realizados na rotina da APS. Nesse sentido, já elaboramos algumas questões que poderão conduzir futuras investigações: Como os profissionais da APS podem analisar sua realidade local e assim estabelecer métodos para abordar os pacientes com pé diabético? Qual o tipo de abordagem causa maior impacto na educação, promoção e prevenção na vida dos pacientes com pé diabético no contexto da APS?

\section{Agradecimentos}

Agradecemos a todos que direta ou indiretamente contribuíram para a realização e sucesso do artigo. 


\section{Referências}

Becker, R. M., Heidemann, I. T. S. B., Meirelles, B. H. S., Costa, M. F. B. N. A. D., Antonini, F. O., \& Durand, M. K. (2018). Práticas de cuidado dos enfermeiros a pessoas com Doenças Crônicas Não Transmissíveis. Revista Brasileira de Enfermagem, 71, $2643-2649$.

Bousquat, A., Giovanella, L., Fausto, M. C. R., Medina, M. G., Martins, C. L., Almeida, P. F., ... \& Mota, P. H. D. S. (2019). A atenção primária em regiões de saúde: política, estrutura e organização. Cadernos de Saúde Pública, 35, e00099118.

Brutti, B., Flores, J., Hermes, J., Martelli, G., da Silva Porto, D., \& Anversa, E. T. R. (2019). Diabete Mellitus: definição, diagnóstico, tratamento e mortalidade no Brasil, Rio Grande do Sul e Santa Maria, no período de 2010 a 2014. Brazilian Journal of Health Review, 2(4), $3174-3182$.

Câmara, S. A. V., Barbosa, T. S., Olivon, V. C., Fernandes, A. L. P., \& Câmara, J. V. (2019). Avaliação do risco para desenvolvimento de diabetes mellitus tipo 2 Em Estudantes Universitários. Revista Ciência Plural, 5(2), 94-110.

Chan, C. B., Dmytruk, K., Labbie, M., \& O’Connell, P. (2020). Organizational changes in diabetic foot care practices for patients at low and moderate risk after implementing a comprehensive foot care program in Alberta, Canada. Journal of foot and ankle research, 13, 1-15.

Chaves, M. A. A., Santos, R. F. D., Moura, L. K. B., Lago, E. C., Sousa, K. H. J. F., \& Almeida, C. A. P. L. (2021). Elaboração e validação de um álbum seriado para prevenção do pé diabético. Revista Cuidarte, 12(1).

Crossetti, M. D. G. O. (2012). Revisão integrativa de pesquisa na enfermagem o rigor cientifico que lhe é exigido. Revista gaúcha de enfermagem, 33(2), 8-9.

Cubas, M. R., Santos, O. M. D., Retzlaff, E. M. A., Telma, H. L. C., Andrade, I. P. S. D., Moser, A. D., \& Erzinger, A. R. (2013). Pé diabético: orientações e conhecimento sobre cuidados preventivos. Fisioterapia em movimento, 26, 647-655.

Daly, B., Arroll, B., Nirantharakumar, K., \& Scragg, R. K. R. (2020). Improved foot management of people with diabetes by primary healthcare nurses in Auckland, New Zealand. NZ Med J, 39-50.

da Silva, G. C. G. V., da Silva, M. A. M., Nogueira, P. P., \& Barbosa, O. L. C. (2021). Desafios da Política Nacional de Atenção Básica à Saúde. Revista PróuniverSUS, 12(1), 60-65.

de Medeiros, L. S. P., Pacheco, R. F., de Medeiros, M. A., \& da Silva, R. M. (2021). O papel do cuidado com as Doenças Crônicas não Transmissíveis na Atenção Primária em Saúde: um olhar da Antropologia da Saúde. Research, Society and Development, 10(12), e267101220250-e267101220250.

de Sousa, N. A., da Silva Lima, J., Teixeira, T. C., Linhares, C. B., Montes, J. V. L., \& Marques, J. V. S. (2019). Fatores de risco e complicações em diabéticos/hipertensos cadastrados no hiperdia. SANARE-Revista de Políticas Públicas, 18(1).

do Nascimento, J. W. A., de Jesus, S. B., Silva, E. C. S., Junior, M. L. F., \& Miranda, A. P. (2019). Neuropatia do pé diabético em usuários de uma unidade de saúde da família. Nursing (São Paulo), 22(256), 3165-3168.

Fonseca, K. P., \& Abi Rached, C. D. (2019). Complicações do diabetes mellitus. International Journal of Health Management Review, 5(1).

Kolltveit, B. C. H., Gjengedal, E., Graue, M., Iversen, M. M., Thorne, S., \& Kirkevold, M. (2016). Telemedicine in diabetes foot care delivery: health care professionals' experience. BMC health services research, 16(1), 1-8.

Malta, D. C., Silva, M. M. A. D., Moura, L. D., \& Morais, O. L. D. (2017). A implantação do Sistema de Vigilância de Doenças Crônicas Não Transmissíveis no Brasil, 2003 a 2015: alcances e desafios. Revista Brasileira de Epidemiologia, 20, 661-675.

Massuda, A. (2020). Mudanças no financiamento da Atenção Primária à Saúde no Sistema de Saúde Brasileiro: avanço ou retrocesso? Ciência \& Saúde Coletiva, 25, 1181-1188.

Matricciani, L., \& Jones, S. (2015). Who cares about foot care? Barriers and enablers of foot self-care practices among non-institutionalized older adults diagnosed with diabetes: an integrative review. The Diabetes Educator, 41(1), 106-117.

Mehndiratta, A., Mishra, S. C., Bhandarkar, P., Chhatbar, K., Cluzeau, F., \& PrimaryCareDoctors, T. (2020). Increasing identification of foot at risk of complications in patients with diabetes: a quality improvement project in an urban primary health centre in India. BMJ open quality, 9(3), e000893.

Medina, M. G., Giovanella, L., Bousquat, A., Mendonça, M. H. M. D., \& Aquino, R. (2020). Atenção primária à saúde em tempos de COVID-19: o que fazer?. Cadernos de Saúde Pública, 36, e00149720.

Mendes, A., \& Funcia, F. O. (2016). SUS e seu financiamento. Marques RM, Piola SF, Roa AC, Ocké-Reis CO, Funcia FR, et al. Sistema de saúde no Brasil: organização e financiamento. Brasília, DF: ABRES, 139-68.

Mendes, Á., Leite, M. G., \& Carnut, L. (2020). Uma metodologia para rateio dos recursos federais do SUS: o índice de necessidades de saúde. Revista de Saúde Pública, 54.

Miranda, G. M. D., Mendes, A. D. C. G., \& Silva, A. L. A. D. (2017). O desafio da organização do Sistema Único de Saúde universal e resolutivo no pacto federativo brasileiro. Saúde e Sociedade, 26, 329-335.

Mullan, L., Wynter, K., Driscoll, A., \& Rasmussen, B. (2021). Barriers and enablers to providing preventative and early intervention diabetes-related foot care: a qualitative study of primary care healthcare professionals' perceptions. Australian Journal of Primary Health.

Nunes, F. M., Sotero, R. F. T. M., Magalhães, T. A., de Quadros, A. C. V. C., \& Oliva, H. N. P. (2019). Prevalência de lesões em órgãos-alvo em diabéticos tipo 2. Revista da Sociedade Brasileira de Clínica Médica, 17(2), 85-89. 
Research, Society and Development, v. 10, n. 15, e161101522608, 2021

(CC BY 4.0) | ISSN 2525-3409 | DOI: http://dx.doi.org/10.33448/rsd-v10i15.22608

Oliveira, I. S. B., Rosa, W. D. A. G., de Oliveira Esequiel, L. I. P., Ferreira, G. M. F., Silva, T. U. M., \& Silva, A. C. P. (2020). Pé diabético: estratégia de prevenção na atenção primária. Revista de Iniciação Científica da Libertas, 10(1), 20.

de Oliveira, R. A., Pires, J. M., Viana, L. G., da Cruz Alencar, M. M. S., Cavalcante, J. V. M. S., Ribeiro, S. G., ... \& Castro, R. C. M. B. (2021). Validação clínica de tecnologia educativa sobre prevenção do pé diabético. Revista Eletrônica Acervo Saúde, 13(1), e5318-e5318.

Parker, C. N., Shuter, P., Maresco-Pennisi, D., Sargent, J., Collins, L., Edwards, H. E., \& Finlayson, K. J. (2019). Implementation of the Champions for Skin Integrity model to improve leg and foot ulcer care in the primary healthcare setting. Journal of clinical nursing, 28(13-14), 2517-2525.

Ramirez-Perdomo, C., Perdomo-Romero, A., \& Rodríguez-Vélez, M. (2019). Conhecimentos e práticas para a prevenção do pé diabético. Revista Gaúcha de Enfermagem, 40.

Rocha-Brischiliari, S. C., Agnolo, C. M. D., Gravena, A. A. F., Lopes, T. C. R., Carvalho, M. D. B., \& Pelloso, S. M. (2014). Doenças crônicas não transmissíveis e associação com fatores de risco. Rev Bras Cardiol, 27(1), 35-42.

Santos, L. (2017). Região de saúde e suas redes de atenção: modelo organizativo-sistêmico do SUS. Ciência \& Saúde Coletiva, 22, 1281-1289.

Sellera, P. E. G., Pedebos, L. A., Harzheim, E., Medeiros, O. L. D., Ramos, L. G., Martins, C., \& D’Avila, O. P. (2020). Monitoramento e avaliação dos atributos da Atenção Primária à Saúde em nível nacional: novos desafios. Ciência \& Saúde Coletiva, 25, 1401-1412.

Schoen, D. E., Gausia, K., Glance, D. G., \& Thompson, S. C. (2016). Improving rural and remote practitioners' knowledge of the diabetic foot: findings from an educational intervention. Journal of foot and ankle research, 9(1), 1-11.

Souza Júnior, E. V. D., Jesus, M. A. S. D., Lapa, P. S., Cruz, J. S. D., Maia, T. F., Barros, V. S., ... \& Boery, E. N. (2019). Internações, óbitos e custos hospitalares por diabetes mellitus. Rev. enferm. UFPE on line, 1-9. 